\title{
Comparison of Conventional Urogram and Magnetic Resonance Urography in Dilated Ureter
}

\author{
PREM KUMAR CHIDAMBARAM ${ }^{1}$, MANIBHARATHI MAYAVAN ${ }^{2}$, S VINAYAGAM $^{3}$, SENTHIL KUMAR AIYAPPAN ${ }^{4}$
}

\section{ABSTRACT}

Introduction: Abdominal pain due to urinary tract obstruction is one of the common problems seen in patients seeking medical attention. Diagnosis of hydronephrosis predominantly depends on X-Ray radiography, contrast Intravenous urogram (IVU), ultrasound and Computed Tomographic (CT) examination. However, IVU is inappropriate for pregnant women and young patients, due to radiation effects and ultrasound examination for ureter is difficult in pregnant abdomen. Hence, Magnetic Resonance Imaging (MRI) urogram (MRU), which is safer, can be a better alternative.

Aim: To compare contrast IVU and MRU in patients with dilated ureter to identify the better modality of choice for urinary tract evaluation in situations where radiation has to be avoided.

Materials and Methods: One hundred and twenty patients with 139 dilated ureters due to 125 causes which include renal calculi, extrinsic compression of pelvic mass, stricture etc. underwent both IVU and MRU. IVU was done with $800 \mathrm{~mA}$ (Milli Ampere) $X$-ray machine with the administration of intravenous non-ionic iodinated contrast agent and MRU was performed on a 1.5 Tesla MRI scanner. The presence, level, grade and cause of ureter dilatation on each modality were interpreted and compared using cross-tabulation with the final diagnosis based on other appropriate modalities including imaging, intraoperative and histopathologic diagnosis for each individual.

Results: The sensitivity of IVU and MRU in detecting hydronephrosis were $99.28 \%$ and $98.65 \%$, respectively. In detecting the level of obstruction, the sensitivity of IVU and MRU were $87.05 \%$ and $96.4 \%$, respectively. The correct diagnosis was made in $64.8 \%$ of the cases by IVU and in $80.8 \%$ of the cases by MRU.

Conclusion: Static MRU is lacking behind IVU in the grading of dilated ureters with a lower grade of hydronephrosis and in assessing the renal function but have a good agreement with IVU in the grading of hydronephrosis in dilated ureters with higher grades. MRU is superior to IVU in the determination of the level and cause of ureteric obstruction. So, IVU can be the first line of investigation in hydroureteronephrosis due to its easy availability and lesser cost and static MRU can be used in situations when the exact cause as to why the ureter is dilated is not clearly defined by IVU and also in circumstances when IVU is contraindicated in patients, like in those with contrast reactions, pregnant patients and also in evaluation of dilated ureter in patients with non functioning kidneys.

Keywords: Diagnostic imaging, Hydronephrosis, Magnetic resonance imaging, Urinary calculi, Urography

\section{INTRODUCTION}

A dilated ureter (hydroureteronephrosis) is one of the common abnormal finding seen in patients seeking medical attention due to various causes, which can be obstructive and non obstructive, congenital and acquired ones, like ectopic ureter, duplex collecting system, retrocaval ureter, ureterocoele, ureteric calculus, infectious diseases, stricture, trauma or urinary structure, non-obstructive causes like vesicoureteric reflux, neurogenic bladder etc., [1]. The dilated ureter is otherwise described as hydronephrosis when there is renal pelvicalyceal dilatation and hydroureteronephrosis when ureter also appears dilated. Usually, these individuals present with urologic complaints like abdomen pain, haematuria (blood in urine), pyuria (pus in urine), dysuria (pain during urination), increased frequency of micturition, etc., to the emergency department.

The accurate diagnosis is vital in planning the appropriate treatment which is the mainstay in the investigation of obstructive and nonobstructive uropathy in patients with dilated ureter [2,3]. IVU helps in evaluation of renal anatomy and functions. IVU however, is inappropriate for pregnant women and young, due to radiation effects, it can also accentuate contrast nephrotoxicity in dehydrated diabetics, renal failure and myeloma patients and more likely could lead to hypersensitivity reactions [4]

CT urography is the gold standard in the evaluation of kidneys, ureter and urinary bladder, which carries the risk of radiation [5]. MRI urogram has also been proven to be equally efficient in diagnosing urinary tract pathologies [6]. "MRU: static fluid imaging", is referred to by some researchers as "hydrographic contrast wherein static or slow-flowing fluids in the body are observed as high-signal-intensity, in contrast to a dark background with very low signal intensity' [7]. In the current study, Static-fluid MRU technique was used with highmagnetic-field (1.5 Tesla) MRI and compared with IVU in patients with all possible causes of dilated ureter. Thereby, identifying the investigation of choice for ureteric obstruction, hence safer MRI Urogram can be the chosen depending on clinical situations. With this background the present study was conducted to compare contrast IVU and MRU in patients with dilated ureter to identify the better modality of choice for urinary tract evaluation in situations where radiation has to be avoided.

\section{MATERIALS AND METHODS}

This was a prospective comparative study done between July 2015 to Aug 2018 in the Department of Radiology, SRM Medical College Hospital, Kattankulathur, Kancheepuram district, Tamil Nadu, India. Institutional ethical committee approval (No.600/IEC) and informed consent from the participants were obtained. This study was carried out in 120 patients (139 ureters-101 unilateral and 19 bilateral ureters) with the dilated ureter, using ultrasound, plain CT or Contrast CT carried out for urologic complaints like abdomen pain, haematuria, pyuria, dysuria, increased frequency of micturition, etc. All age groups and both sexes presented to radiology department with urologic complaints were included in the study. Patients who had 
contraindications for IVU (patients with the previous history of Contrast Reactions, patients with abnormal renal function, pregnant patients) or MRU (patients with cardiac pacemakers, metallic implants) and patients who were not willing to give consent were excluded. Both the studies were performed on the same day in the majority of the participants, sparing few, for whom both studies were completed within a week. Clinical history was taken and IVU was performed ensuring an overnight fasting and normal serum creatinine level.

The IVU study was done with 800mA X-Ray machine. Standard IVU procedure of anteroposterior scout radiograph of KUB, followed by 3 survey images at 5, 15, and 30 minutes taken after intravenous injection of bolus ( $1 \mathrm{ml} / \mathrm{kg}$ for adults and $1.5 \mathrm{ml} / \mathrm{kg}$ for children) of nonionic iodinated contrast agent, lohexol (Omnipaque 300; GE Healthcare, Milwaukee, WI). Emergency medicines to treat contrast reactions were readily made available. None of the patients included in the current study developed such adverse reactions. In cases of delayed excretion, delayed images were taken at 24 hours.

$\mathrm{MRI}$ was performed with the patient in the supine position in a 1.5T Magnetom ESSENZA scanner (Siemens, Erlangen, Germany) using a phased-array body coil. The MR examination was conducted without specific preparatory measures, such as fasting or drinking. The pulse sequences used are shown in [Table/Fig-1], which took 15-30 minutes for each study. Additional sequences were done according to the need in each patient. Contrast agent was not used. Post processing was performed using a Maximum Intensity Projection (MIP) algorithm in parallel with the long axis of the urinary tract.

Both IVU and MRU studies were interpreted for the presence of ureter dilatation level and grade of hydronephrosis and the most likely cause for dilatation. Level of dilatation was described like pelvi ureteric junction, proximal, mid and lower ureter and vesicoureteric junction [8], where proximal segment is from pelviureteric junction to sacrum level, mid is at the sacrum level and distal is from the sacrum to vesicoureteric junction level. Grading of hydronephrosis was done based on the dilatation of renal fornix, loss of papillary impression and calyceal ballooning as increasing grades, like mild blunting of fornices as 1, calyceal dilatation and preserved papillary indentation as 2, Round calyx and loss of papillary indentation as 3 and extreme calyceal ballooning as 4 [5]. The cause of obstruction was confirmed with appropriate available imaging, surgical or histopathological data depending on the clinical situation and follow-up.

\section{STATISTICAL ANALYSIS}

Cross-tabulation derived from data was used for calculation of sensitivity and specificity. The sensitivity of MRU was calculated using IVU as the standard of reference by using commercially available software (SPSS 15; SPSS, Chicago, IL). Accuracy of IVU and $\mathrm{MRU}$ for diagnosing the cause of the obstruction was compared with the final diagnosis.

\section{RESULTS}

Among the total of 120 patients, 19 had bilateral ureteral dilatation, so the total dilated ureters studied for obstruction and grading were 139. The age ranged from 3 to 75 years with mean as 37.74 years and highest incidence were found in 31-40 years age group (35\%) and the males outnumbered females by $\sim 100 \%$.

\section{Comparison of Grade of Hydronephrosis:}

In detecting the Grade of Hydronephrosis, of the total of 139, 138 ureters were detected as dilated by IVU. Then, by keeping IVU as the standard for these 138 ureters, MRU findings were compared with IVU and the sensitivity for Grading of Hydronephrosis by MRU was determined. There were 122 Ureters in MRU that had similar grading by IVU and 16 ureters were either under or over graded.

Among these, in Grade 0 of IVU, there were a total of 6 Ureters, of which only 2 Ureters had similar grading by MRU. Two ureters were not detected as dilatation at all by MRU and interpreted as normal, and the other 2 ureters were upgraded by one Grade to Grade 1. Hence, the Sensitivity of MRU for Grade 0 was 33.33\%. In 18 grade 1 ureters detected as by IVU, 16 ureters had similar gradings with MRU and only 2 ureters were upgraded by one. So, the Sensitivity of MRU for Grade 1 was 88.88\%; Among 68 Grade 2 hydronephrosis by IVU, 63 ureters had similar MRU gradings, 3 ureters were upgraded by one and 2 ureters were downgraded by one. So, the Sensitivity of MRU for Grade 2 was $92.67 \%$. Of the 44 grade, 3 ureters by IVU, 39 ureters of MRU had similar gradings and 5 were upgraded by one. Hence, the sensitivity of MRU for Grade 3 was $88.63 \%$ and 2 ureters were accurately detected as Grade 4 by IVU and MRU [Table/Fig-2]. In addition to this, there was one obstruction that was not detected at all in IVU and found to have Grade 4 Hydronephrosis by MRU. Therefore the overall sensitivity of MRU was found to be $88.40 \%$.

Among the 19 patients who had dilatation of both the ureters, 14 had a common cause of dilatation of bilateral ureters like stricture, calculi on bilateral ureter or urinary bladder outlet obstruction and the other five patients had separate causes for ureteral dilatation on each side [Table/Fig-3]. So the total number of causes were $125(120+5)$ [Table/Fig-4]. Pain (75\%) was the predominant presenting symptom, followed by haematuria (5\%), dysuria (5\%) and fever (3\%). Right lumbar and iliac region pain was the most common site of abdominal pain and right side (47\%) has a higher incidence than the left (36\%).

\section{Detection of Dilatation of Ureter}

Ureter dilatation was detected in 138 Ureters by IVU and 137 by $\mathrm{MRU}$, so the sensitivity of IVU and MRU in the detection of dilatation of ureter is 99.28 and 98.65 , respectively.

\section{Comparison of the Level of Obstruction}

Of the 139 dilated ureters, level seen at proximal third in 25 ureters, 19 ureters in mid-third using IVU and MRU; was in distal third for 78 ureters in IVU and 66 ureters by MRU; was in the VUJ for 7 ureters by IVU and 20 ureters by MRU and was in the level of bladder for 4 ureters in both the modalities. For 6 ureters in IVU and 5 ureters in $\mathrm{MRU}$, the exact level at which ureter was dilated was undetermined. After correlating IVU with $\mathrm{MRU}$, there were a total of 18 ureters were not similar in level of ureter dilatation. Among these, 12 were misinterpreted as distal third by IVU to be the site of obstruction on CT correlation, which was found to be in the VUJ and was correctly interpreted as VUJ obstruction by MRU. So, the overall sensitivity to accurately predict the level of obstruction was by IVU and MRU was $87.05 \%$ and $96.4 \%$, respectively. The most common level at which Ureter is dilated was found to be Distal ureter in both IVU and MRU [Table/Fig-3].

\begin{tabular}{|c|c|c|c|c|c|c|c|}
\hline Sequence & TR (ms) & $\mathrm{TE}_{\text {eff }}(\mathrm{ms})$ & No. of slice & Thickness (mm) & Gap (mm) & Matrix & $\mathrm{FOV}(\mathrm{mm})$ \\
\hline Localizer & 150 & 4.8 & 3 & 8 & 4 & $128 \times 256$ & 400 \\
\hline T2W HASTE-FS (coronal thick slab) & 2800 & 1100 & 1 & $40-80$ & - & $256 \times 256$ & 360 \\
\hline T2W HASTE-FS (coronal thin slab) & 18 & 95 & $30-40$ & 5 & 4 & $256 \times 256$ & 360 \\
\hline T2W 3D FSE (coronal rsta ${ }^{a}$ & 4000 & 400 & $40-50$ & 2 & 2 & $179 \times 256$ & 360 \\
\hline T2W TRUFI (axial bh) & 3.57 & 1.51 & 50 & 4 & $-\mathrm{b}$ & $192 \times 256$ & $340-380$ \\
\hline T2W TRUFI (coronal bh) & 3.57 & 1.51 & 20 & 4 & 6 & $240 \times 256$ & 360 \\
\hline
\end{tabular}




\begin{tabular}{|c|c|c|c|c|}
\hline \multirow[t]{2}{*}{ Grade of hydronephrosis } & \multicolumn{2}{|c|}{ IVU } & \multicolumn{2}{|c|}{ MRU } \\
\hline & $\begin{array}{c}\text { No.of } \\
\text { ureters }\end{array}$ & Percentage & $\begin{array}{c}\text { No.of } \\
\text { ureters }\end{array}$ & Percentage \\
\hline 0 & 6 & 4.3 & 2 & 1.4 \\
\hline 1 & 18 & 12.9 & 20 & 14.4 \\
\hline 2 & 68 & 48.9 & 65 & 46.8 \\
\hline 3 & 44 & 31.7 & 42 & 30.2 \\
\hline 4 & 2 & 1.4 & 8 & 5.8 \\
\hline Undetected & 1 & 0.7 & 2 & 1.4 \\
\hline Total & 139 & 100 & 139 & 100 \\
\hline
\end{tabular}

\begin{tabular}{|l|c|c|c|c|}
\hline Level of dilatation & \multicolumn{2}{|c|}{ IVU } & \multicolumn{2}{c|}{ MRU } \\
\hline & No.of ureters & Percentage & No. of ureters & Percentage \\
\hline Proximal Ureter & 25 & 18 & 25 & 18 \\
\hline Mid Ureter & 19 & 13.7 & 19 & 13.7 \\
\hline Distal Ureter & 78 & 56.1 & 66 & 47.5 \\
\hline VUJ & 7 & 5 & 20 & 14.4 \\
\hline Bladder & 4 & 2.9 & 4 & 2.9 \\
\hline Undetected & 6 & 4.3 & 5 & 3.6 \\
\hline Total & 139 & 100 & 139 & 10 \\
\hline
\end{tabular}

[Table/Fig-3]: Comparison of level at which ureter is dilated by IVU and MRU.

\begin{tabular}{|l|c|c|c|c|}
\hline Causes of dilatation & \multicolumn{2}{|c|}{ IVU } & \multicolumn{2}{c|}{ MRU } \\
\hline & No & Percentage & No & Percentage \\
\hline Calculus & 57 & 45.6 & 73 & 58.4 \\
\hline Pelvic mass & 9 & 7.2 & 9 & 7.2 \\
\hline Stricture & 5 & 4 & 5 & 4 \\
\hline Retrocaval ureter & 3 & 2.4 & 3 & 2.4 \\
\hline Bilateral duplex collecting system & 1 & 0.8 & 1 & 0.8 \\
\hline Ectopic ureter with ureterocele & 1 & 0.8 & 1 & 0.8 \\
\hline Cystitis & 2 & 1.6 & 2 & 1.6 \\
\hline Neurogenic bladder & 2 & 1.6 & 2 & 1.6 \\
\hline Others & 1 & 0.8 & 5 & 4 \\
\hline Undetected & 44 & 35.2 & 24 & 19.2 \\
\hline Total & 125 & 100 & 125 & 100 \\
\hline
\end{tabular}

[Table/Fig-4]: Detection of Cause of dilatation of Ureter by IVU and MRU.

\section{Detection of Cause of Dilatation of Ureter}

In detecting the cause of ureteral dilatation, IVU and MRU had a sensitivity of $64.8 \%$ and $80.8 \%$, respectively. The most common cause of dilatation of ureter was ureteral calculi followed by pelvic masses. IVU could detect larger calculi (almost all the calculi over $7 \mathrm{~mm}$ in size and a few calculi of $6-5 \mathrm{~mm}$ in size) accurately and many smaller calculi in $6-5 \mathrm{~mm}$ range and $<5 \mathrm{~mm}$ calculi were not detected by IVU. In MRI, the large calculi and smaller calculi in the $6-4 \mathrm{~mm}$ range were also diagnosed, thereby having a higher sensitivity in diagnosing calculi. However, very small and tiny calculi of sizes $3 \mathrm{~mm}$ or lesser were not diagnosed on MRU [Table/Fig-4].

\section{DISCUSSION}

Although IVU has been the mainstay in the evaluation of hydronephrosis to assess the level and grade of obstruction and excretory function of the kidneys, MRU offers the possibility of evaluating the urinary system showing high-quality images of the urinary tract without exposing to radiations. The usefulness of MRU using heavily T2-weighted sequences has been discussed in a few reports $[9,10]$.

Even though there is excellent sensitivity of IVU and MRU in detecting dilated ureter, two ureters not detected in MRU were of Grade 0 hydronephrosis, due to Grade 1 vesicoureteric reflux and one ureter in IVU due to a non-function upper moiety of double

collecting system with double ureters and ectopic ureterocele [Table/Fig-5]. There is a slight edge of IVU (99.28\%) over MRU $(98.65 \%)$ in terms of detection of the dilated ureter. However, if there were non-functioning kidneys with hydronephrosis and less Grade 0 hydroureteronephrosis in a study, then probably the sensitivity of MRU would be much higher than IVU.

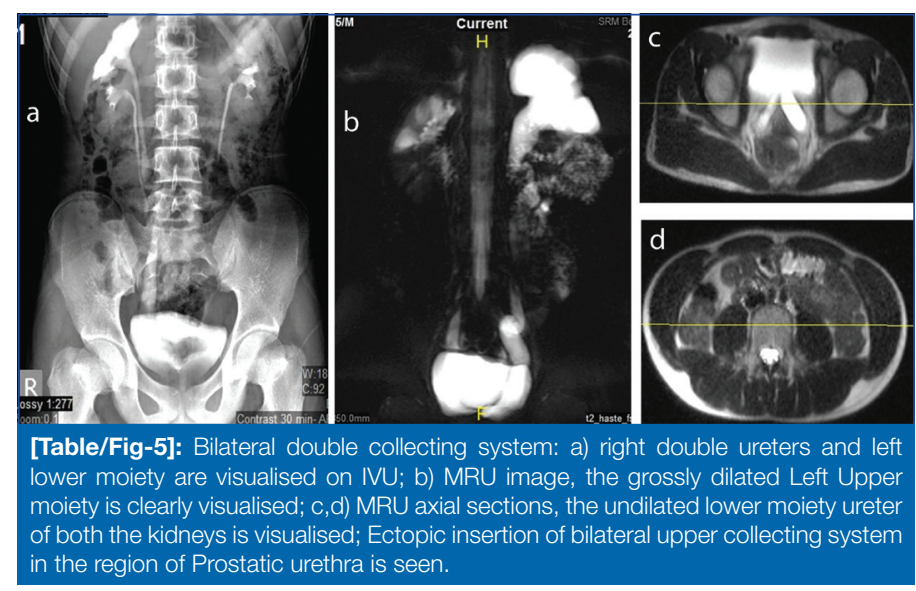

Khanna PC et al., and Muthusami P et al., did a similar study and showed the importance of MRU and showed an MRU sensitivity of $95 \%[9,10]$. The slightly reduced sensitivity is likely due to a large proportion of cases with a low grade of hydronephrosis, nearly two-thirds $(66.18 \%)$ of ureters being grade 2 or less. Louca G et al., showed a sensitivity of $100 \%$ in detecting hydronephrosis by MRU, carried out in 33 Patients [11]. Mild decrease in sensitivity in comparison to this is probably due to the less number of cases than the current study of 120 patients. Nolte-Ernsting CC et al., in a study using diuretic and contrast-enhanced MRU, showed the sensitivity of MRU to be $90 \%$ [12]. This too had a good correlation with current study in terms of detecting ureter dilatation by MRU.

In detecting the Grade of hydronephrosis, from a total of 139 ureters, 138 ureters were detected to be dilated by IVU. There were 122 ureters in MRU that had similar grading by IVU and 16 ureters that were either under or over graded. In addition to this, there was one unit that was not detected at all in IVU and found to have Grade 4 hydronephrosis by MRU. Therefore the overall sensitivity of $\mathrm{MRU}$ was found to be $88.40 \%$.

The lesser distension of pelvicalyceal systems and ureters in lower grades of hydronephrosis causes poorer assessment by MRU, which were downgraded. Specifically, 4 cases (2.89\%) of the total were downgraded, all of these being interpreted as one grade lower. On the other hand, 12 cases (8.68\%) of the total were upgraded by $\mathrm{MRU}$ and all of these being interpreted as one Grade higher.

Louca G et al., Hussain S et al., and Regan F et al., reported to have $100 \%$ sensitivity for locating and grading the obstruction $[11,13,14]$. Here, overall sensitivity was $88.40 \%$ in Grading of hydronephrosis, probably due to the larger number of cases and proportionately lower grades of hydronephrosis (Grade 0,1 or 2). There is lesser sensitivity of MRU for lower grades and increased sensitivity for higher grades as observed by Muthusami P et al., [10].

It has also been found that static MRU is lacking behind IVU in the evaluation of mild ureter dilatation (grades 0,1 and 2). This is due to calyceal, forniceal, and infundibular anatomy, which cannot be seen with the same detail in static MRU as in excretory urography [15]. In this context, it has been found that the use of a paramagnetic contrast agent improves the diagnostic value of MRU. Nevertheless, current aim was to delineate the role of a non-contrast method.

Overall good sensitivity to accurately predict the level of ureter obstruction by IVU and MRU, MRU had slightly higher sensitivity than IVU, probably due to multiplanar images. Louca $\mathrm{G}$ et al., and Catalano C et al., showed $100 \%$ sensitivity in the detection of the level of obstruction $[11,16]$. The current study also correlated well 
with these studies, but with comparatively lesser sensitivity, probably due to the larger group $(n=139)$ in our study in contrast to lesser numbers ( $\mathrm{n}=25$ and 43$)$.

In detecting the cause of dilatation of ureter, IVU and MRU had a sensitivity of $64.8 \%$ and $80.8 \%$ respectively. Smaller calculi produce reduced density to appear radio-opaque, to be distinctly appreciated by Conventional Radiograph or Conventional Excretory Urography. On the other hand in MRU, calculi appear as flow voids. The smaller calculi in the 6-4 $\mathrm{mm}$ range were also diagnosed, thereby having a higher sensitivity in diagnosing calculi in comparison to IVU. However, very small and tiny calculi of sizes $3 \mathrm{~mm}$ or lesser were not diagnosed on MRU. The reason for this is probably due to poor spatial resolution as a result of mild body motion and lack of respiratory gating with timeconsuming scan procedure.

The other causes that were not diagnosed on IVU were mainly extraluminal causes that included a case of duodenal diverticulitis with abscess formation, right inguinal hernia-post-op status with ureter as contents, deposit near left common lliac artery and a case of right retroperitoneal mass causing ureteral obstruction with proximal dilatation. These were diagnosed by MRU and so MRU significantly scores over IVU in detecting extra luminal aetiologies because of its multiplanar imaging facility. Added to it, MRU also helps in studying the complete extension of the lesion and in tracing the non dilated ureter even distal to the site of obstruction.

In 9 patients, by IVU ureter dilatation was detected and diagnosed to be due to large pelvic masses, however, only by MRI we could specifically determine whether they were ovarian, paraovarian cysts or uterine fibroids [Table/Fig-6]. IVU and MRU scored equally in diagnosing cystitis, neurogenic bladder and retrocaval ureter. There were 5 cases shown to have mild ureter dilatation with Grade 0 hydronephrosis in both IVU and MRU. However, the cause was not detected in both these modalities. Later, it was diagnosed with Mild Vesicoureteric Reflux by MCU.

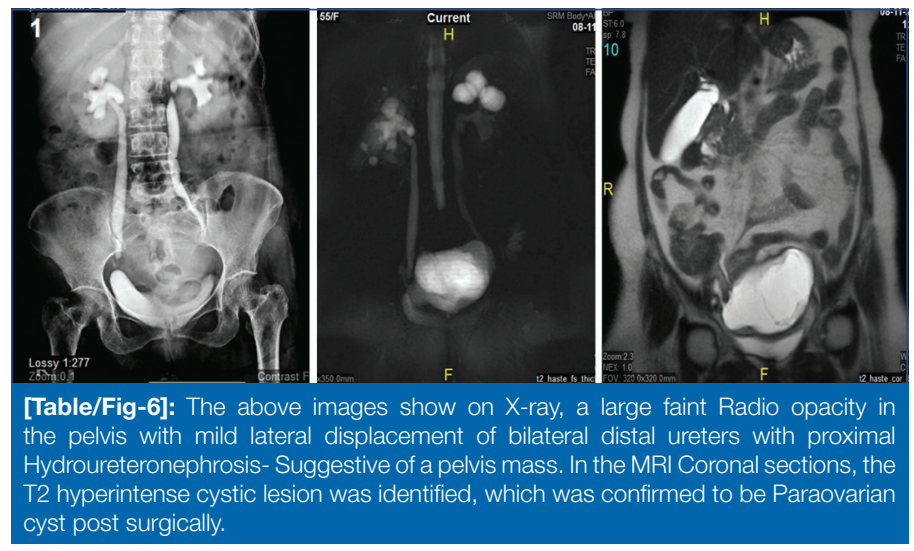

Muthusami $P$ et al., showed in a study of 69 patients, a sensitivity of IVU and $\mathrm{MRU}$ in detecting the cause of ureteral obstruction as $89.2 \%$ and $93.8 \%$, respectively [10]. In the current study, sensitivity for IVU and MRU are $64.8 \%$ and $80.8 \%$, respectively, ours too showed a higher sensitivity for MRU in comparison with IVU. The large difference in the sensitivity between the previous study and current study was due to a large number of small calculi not being diagnosed in MRU and the higher number being undiagnosed in IVU. If, we had omitted a large portion of these tiny ureteric calculi we could have had a higher sensitivity in the current study.

Emad-Eldin S et al., and Jung $P$ et al., compared IVU and MRU, showed a diagnostic accuracy of $63.4 \%$ and $86.5 \%$ in IVU and $\mathrm{MRU}$ respectively, MRU scored over IVU in the detection of both calculi and non-calculi causes $[17,18]$. The current study correlated well with this study in having a sensitivity of $64.8 \%$ and $80.8 \%$ for IVU and MRU respectively in which, both in the detection of Calculi and non-calculi cause.
Unlike Louca G et al., showed a sensitivity of (48\%) in diagnosing the underlying cause, the current study showed much higher sensitivity [11]. There is disagreement between authors regarding the ability of IVU and MRU to detect the cause of obstruction. The detection rate varies from 50\%-100\% in different studies [19,20]. Currents study showed the detection rate was $64.8 \%$ and $80.8 \%$ for IVU and MRU respectively, correlating well with the results of similar studies.

There were also a few small and moderate-sized calculi that were better detected in MRU. The difficulty in detection of very small renal and ureteral stones with T2-weighted sequences is a known limitation of MRU and this might be one reason of the high accuracy of diagnosis by MRU in the current study $[6,14]$.

\section{LIMITATION}

Majority of the pelvic mass and other pathologies could not be followed up by histopathological methods. Patients with cardiac pacemakers and aneurysm clips were excluded for MRI. Importance of the study can be further improved by including all spectrums of pathologies causing hydroureteronephrosis and studying more number of patients as multicenter trials.

\section{CONCLUSION}

Static multiplanar MRU provides an image that gives a good understanding of the entire urinary system and extraluminal structures, is very useful in the evaluation of patients with dilated ureter in both functioning and non-functioning kidneys. However, it lacks its ability to evaluate renal function. Although there is administration of intravenous contrast agent which predisposes to the risk of contrast reactions, along with the risk of radiations, IVU is cost-effective, easily available and useful in the evaluation of dilated ureter. It is very much reliable in the evaluation of renal function.

Observation was that in the detection of ureter dilatation, IVU is superior in detecting lower grades (Grade 2 or less), and MRU in superior in detecting higher grades (Grade 3 and 4). MRU shows lesser sensitivity for lower grades of hydronephrosis. MRU was accurate in detecting the level and cause of obstruction, compared to IVU.

So, IVU can be kept as the first line of investigation in patients with dilated ureter due to its easy availability and lesser cost, and static MRU can be used in situations when the exact cause as to why the ureter is dilated is not clearly defined by IVU and also in circumstances when IVU is contraindicated in patients, like in those with contrast reactions, pregnant patients and also in evaluation of dilated ureter in patients with non functioning kidneys.

\section{REFERENCES}

[1] King LR. Megaloureter: Definition, diagnosis and management. J Urol. 1980;123(2):222-23.

[2] Roy C, Ohana M, Host P, Alemann G, Labani A, Wattiez A, et al. MR urography (MRU) of non-dilated ureter with diuretic administration: Static fluid 2D FSE T2weighted versus 3D gadolinium T1-weighted GE excretory MR. Eur J Radiol Open.2014;1:06-13.

[3] Roy C, Saussine C, Guth S, Horviller S, Tuchmann C, Vasilescu C, et al. MR urography in the evaluation of urinary tract obstruction. Abdom Imaging 1998;23(1):27-34.

[4] Macleod MA, Houston AS. A comparison of three methods of assessing renal function. Eur J Nucl Med. 1981;6(5):183-89.

[5] Shine S. Urinary calculus: IVU vs. CT renal stone? A critically appraised topic. Abdom Imaging. 2008;33(1):41-43.

[6] Sudah M, Vanninen RL, Partanen K, Kainulainen S, Malinen A, Heino A, et al Patients with acute flank pain: Comparison of MR urography with unenhanced helical CT. Radiology. 2002;223(1):98-105.

[7] O'Connor OJ, McLaughlin P, Maher MM. MR Urography. AJR Am J Roentgenol. 2010;195(3):W201-06.

[8] Leyendecker JR, Barnes CE, Zagoria RJ. MR urography: Techniques and clinical applications. Radiographics: A review publication of the Radiological Society of North America, Inc. 2008;28(1):23-46; discussion -7.

[9] Khanna PC, Karnik ND, Jankharia BG, Merchant SA, Joshi AR, Kukreja KU Magnetic resonance urography (MRU) versus intravenous urography (IVU) in obstructive uropathy: a prospective study of 30 cases. J Assoc Physicians India. 2005;53:527-34. 
[10] Muthusami P, Bhuvaneswari V, Elangovan S, Dorairajan LN, Ramesh A. The role of static magnetic resonance urography in the evaluation of obstructive uropathy. Urology. 2013;81(3):623-27.

[11] Louca G, Liberopoulos K, Fidas A, Nikolakopoulou Z, Lykourinas M, Strigaris K. MR urography in the diagnosis of urinary tract obstruction. Eur Urol. 1999;35(2):102-08.

[12] Nolte-Ernsting CC, Bucker A, Adam GB, Neuerburg JM, Jung P, Hunter DW, et al. Gadolinium-enhanced excretory MR urography after low-dose diuretic injection: comparison with conventional excretory urography. Radiology. 1998;209(1):147-57.

[13] Hussain S, O'Malley M, Jara H, Sadeghi-Nejad H, Yucel EK. MR urography. Magn Reson Imaging Clin N Am. 1997;5(1):95-106.

[14] Regan F, Bohlman ME, Khazan R, Rodriguez R, Schultze-Haakh H. MR urography using HASTE imaging in the assessment of ureteric obstruction. AJR Am J Roentgenol. 1996;167(5):1115-20.

[15] Hattery RR, King BF. Technique and application of MR urography. Radiology. 1995; 194(1):25-27
[16] Catalano C, Pavone P, Laghi A, Scipioni A, Panebianco V, Brillo R, et al. MR pyelography and conventional MR imaging in urinary tract obstruction. Acta Radiol. 1999;40(2):198-202.

[17] Emad-Eldin S, Abdelaziz O, El-Diasty TA. Diagnostic value of combined static-excretory MR Urography in children with hydronephrosis. J Adv Res. 2015;6(2):145-53.

[18] Jung P, Brauers A, Nolte-Ernsting CA, Jakse G, Gunther RW. Magnetic resonance urography enhanced by gadolinium and diuretics: a comparison with conventional urography in diagnosing the cause of ureteric obstruction. BJU Int. 2000;86(9):960-65.

[19] O'Malley ME, Soto JA, Yucel EK, Hussain S. MR urography: evaluation of a three-dimensional fast spin-echo technique in patients with hydronephrosis. AJR Am J Roentgenol. 1997;168(2):387-92.

[20] Reuther G, Kiefer B, Wandl E. Visualization of urinary tract dilatation: value of single-shot MR urography. Eur Radiol. 1997;7(8):1276-81.

\section{PARTICULARS OF CONTRIBUTORS:}

1. Associate Professor, Department of Radiodiagnosis, SRM Medical College, Chennai, Tamil Nadu, India.

2. Resident, Department of Radiodiagnosis, SRM Medical College, Kanchipuram, Tamil Nadu, India.

3. Professor and Head, Department of Radiodiagnosis, SRM Medical College, Kanchipuram, Tamil Nadu, India.

4. Professor, Department of Radiodiagnosis, SRM Medical College, Kanchipuram, Tamil Nadu, India

NAME, ADDRESS, E-MAIL ID OF THE CORRESPONDING AUTHOR:

Dr. Prem Kumar Chidambaram,

3, Gurudev Flats, 18, Adigalar Salai, NH1, Maraimalai Nagar, Chennai, Tamil Nadu, India.

E-mail: drcpremkumar@gmail.com

Date of Submission: Jun 18, 2019

Date of Peer Review: Jun 28, 2019

Date of Acceptance: Sep 24, 2019

Date of Publishing: Oct 01, 2019 